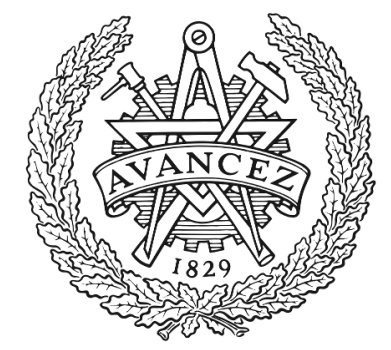

CHALMERS

UNIVERSITY OF TECHNOLOGY

\title{
Prospective new PMC based gap waveguide shielding for microwave modules
}

Downloaded from: https://research.chalmers.se, 2023-04-26 15:12 UTC

Citation for the original published paper (version of record):

Uz Zaman, A., Rajo, E., Kildal, P. (2014). Prospective new PMC based gap waveguide shielding for microwave modules. IEEE International Symposium on Electromagnetic Compatibility, EMC Europe 2014; Swedish Exhibition and Congress CentreGothenburg; Sweden; 1 September 2014 through 4 September 2014: 459-463. http://dx.doi.org/10.1109/EMCEurope.2014.6930950

N.B. When citing this work, cite the original published paper. 


\title{
Prospective New PMC based Gap Waveguide Shielding for Microwave Modules
}

\author{
Ashraf Uz Zaman ${ }^{1}$, Eva Rajo-Iglesias ${ }^{2}$ and Per-Simon Kildal ${ }^{1}$ \\ ${ }^{1}$ Department of Signals and Systems, Chalmers University of Technology, Göteborg, Sweden \\ ${ }^{2}$ Department of Signal Theory and Communications, University Carlos III of Madrid, Madrid, Spain \\ zaman@chalmers.se; eva@tsc.uc3m.es; per-simon.kildal@chalmers.se
}

\begin{abstract}
In this paper we present a general overview of Gap Waveguide shielding or packaging technique applied in various passive or active microwave modules in order to improve the electrical performance of the respective modules. In this new packaging approach, we use periodic structures to achieve a stopband by enforcing cutoff condition for all global parallel-plate modes even within an oversized microwave mechanical assembly. Within this stop-band, we are able to suppress all unwanted cavity modes, substrate modes, unwanted EMI and RF feedback, thereby improving the overall system performance. In this work, we recapitulate several realizations of gap waveguide packaging solution, relevant for both high frequency and low frequency microwave applications.
\end{abstract}

Index Terms - oversized cavity; parallel-plate modes; stopband; substrate modes; cross-talk; imperfect joints.

\section{INTRODUCTION}

The heavy congestion at the existing radio frequency spectrum allocated for the today's wireless communications motivates and accelerates the research work at mmWave bands or even higher frequency range where more spectrum space is available for massive data rate delivery. The inclination of microwave system research and development is shifting towards smaller size, reliable, high-performance and high-yield multifunctional products. As a result, RF engineers are designing various RF components based on system on chip (SOC), system on package (SOP) and substrate integrated circuits (SIC) [1-2]. While designing such components, they are facing extreme challenges such as noise coupling between RF/Digital sections, signal integrity problems, unwanted EM interference to neighboring circuit components and cross-talk problems. For the complete microwave system to function well, the RF engineers must suppress three aspects of EMC problem: emissions, susceptibility and self-compatibility [3]. RF emissions may occur from a specific circuit component and may upset other nearby circuits. On the other hand, external RF energy in the form of feedback or noise coupling may upset performance of a working circuit, leading to susceptibility problem (or a lack of immunity). Finally, energy internal to the system may interfere with other internal circuits, resulting in a self-compatibility problem. Therefore, if not properly controlled, the RF interference or cross-talk can cause each microwave system to malfunction and even fail.
So, one of the most challenging design aspects of RF packaging or shielding is to maintain high isolation among the adjacent microwave circuits or critical components and suppress unwanted EMI [4-5].

Today's microwave engineers are still using traditional multi-chip module (MCM) style where several packaged SMT chips or QFN style MMIC chips are placed on a single carrier substrate. These individual MMICs are connected through simple microstrip lines or coplanar waveguide lines. This is shown in fig.1. This solution consists of using a separate metallic shielding enclosure for each critical component (typically the active components). However, such solutions require lots of vias in the substrate to stop energy coupling via substrate modes and also require lossy absorbers to dampen the high Q cavity modes [6-7]. For this well-known solution, misalignments, material dents, oxidation, and imperfect joints, narrow slots or openings between adjacent sections are the main reasons of shielding failure and generate so-called higher modes due to strange boundary conditions; distort the desired mode of propagation; and also degrade the electrical performance severely. In practice, the effectiveness of this shielding technique goes down with frequency; the higher the frequency, the lower is usually the effectiveness of the technique mentioned here.

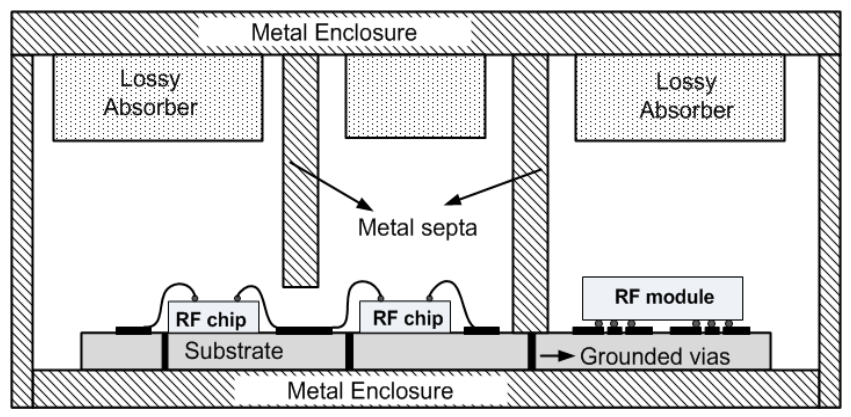

Fig.1 Traditional multi-compartment microwave module

To deal with the above-mentioned issues, we have proposed a new packaging technique for multi compartment microwave modules. The proposed new packaging or shielding technique is actually an extension of the recently developed gap waveguide technology [8-10]. To be more precise, the gap waveguide technology itself is deeply rooted in the concept of hard and soft surface [11]. 


\section{CONCEPT OF GAP WAVEGUIDE PACKAGING OR SHEILDING}

In a microwave module, the MMICs, hybrid circuits, other passive components or interconnect lines may be based on different types of transmission line media such as microstrip lines or coplanar waveguides (CPWs). Published studies in [12-13] show that significant power leakage exists on various printed circuit transmission lines. This leakage occurs in the form of the surface waves or the substrate modes associated with the dielectric substrate, causing serious cross-talk and interference problems. Apart from this problems of substrate modes or surface waves, interconnects and transitions between different components may produce significant spurious radiation or standing waves. This can easily get coupled (over the air) to the neighboring circuit elements resulting in interference and cross-talk. Therefore, we propose a new packaging technique which handles cavity resonances within the metal enclosure, over the air coupling between the adjacent components, and also the coupling via the substrate modes. The concept of the basic gap waveguide technology and gap waveguide packaging is shown in fig. 2 .
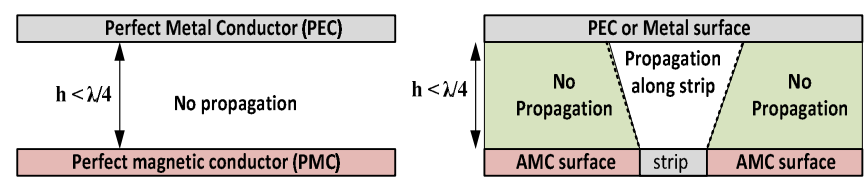

Fig.2(a) Basic operation of Gap waveguide technology.
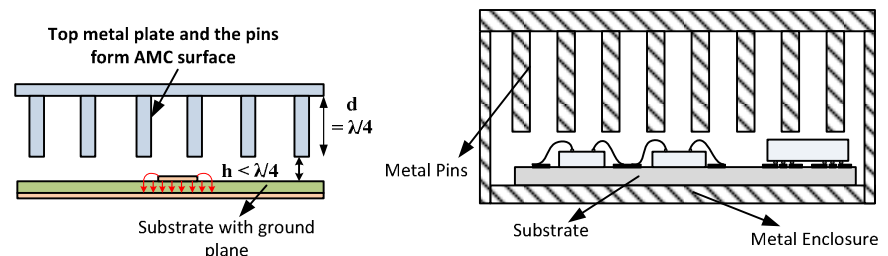

Fig.2(b) New Gap waveguide packaging technology.

As shown in fig.2 (a), this new technology uses the basic cut-off of a PEC-PMC parallel-plate waveguide configuration to control the electromagnetic wave propagation between the two parallel plates. This PEC-PMC configuration creates a cutoff condition for all the parallel-plate modes and no propagation is possible as long as the distance between them is kept smaller than $\lambda / 4$. However, if a PEC strip is placed in the PMC layer, EM wave can propagate along the strip without being leaked into the oversized parallel-plate waveguide. Thus, within the Gap waveguide structures, it is possible to control the desired mode of propagation even though the structure can be over-moded. Recently, many critical microwave components such as filters and antennas have been designed based on this technology [14-15]. Also, the gap waveguide technology is scalable to higher frequencies as silicon based micro-fabrication techniques can be applied for cheap production of mmWave gap waveguide structures [16].The Gap waveguide concept can be easily extended for packaging application and is shown in fig.2 (b). For this application, the ground of the circuit board is ideally the
Perfect Electric Conductor (PEC). The top lid is a specially designed metal lid containing periodic textures and it works as a Perfectly Magnetic Conductor (PMC) within a specific stopband. Thus, we generate a PEC-PMC parallel-plate structure, even in the presence of the dielectric substrate, where all unwanted parallel-plate modes are suppressed within a specific frequency range. The application for gap waveguide packaging was first demonstrated successfully for passive microstrip circuits in [17-19]. In these applications, periodic metal pins were used to emulate the PMC behavior, but there are several other ways to realize the PMC condition. These will be discussed more details in section-IV. The PMC based gap waveguide packaging technique enables inclusion of packaging from the start when designing microwave circuits, instead of struggling with it afterwards [20]. This saves computer time while doing the microstrip circuit simulations as PMC $\left(H_{t}=0\right)$ can be implemented very easily in most of the full-wave EM solvers. Also, ideal PMC can be used as a good first order approximation for Gap waveguide structures giving an extra advantage to this new packaging technique in terms of production time. This is because, packaging effects can be considered already in the simulation phase and there will be no need for cut and trial in the final stage of the product cycle.

\section{GAP WAVEGUIDE PACKAGING FOR ACTIVE MICROWAVE COMPONENTS}

Instability in an amplifier chain is mainly the result of unwanted feedback across the amplifier and such feedback is normally introduced from shielding imperfections. In this present section, we explain how we applied the Gap waveguide packaging technique on a Ka-band high gain amplifier chain having four cascaded units. For manufacturing simplicity, we used periodic metal pins to create the parallelplate stop band. The dispersion diagram for unit cell of the periodic metal pin is shown in fig. 3 .

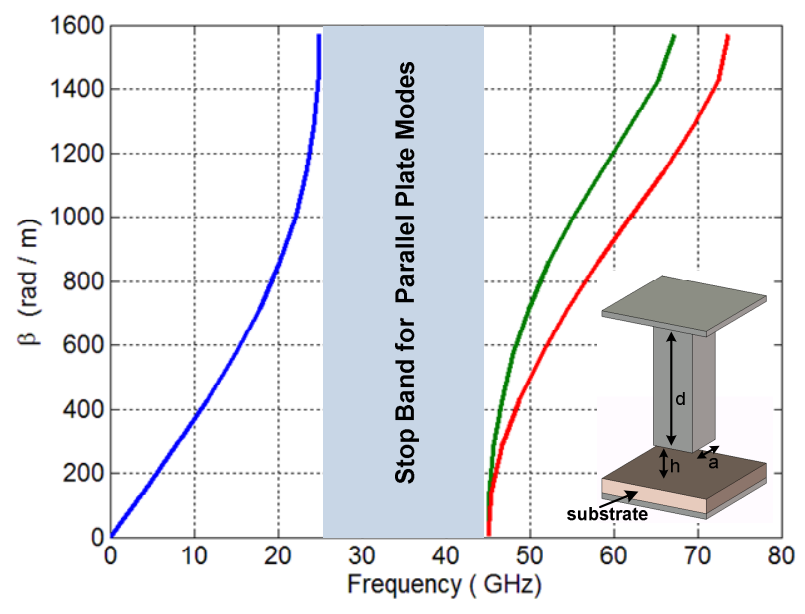

Fig.3 Dispersion diagram for unit cell; $\mathrm{a}=0.8 \mathrm{~mm}, \mathrm{~h}=0.5 \mathrm{~mm}, \mathrm{~d}=2.25 \mathrm{~mm}$.

After obtaining the periodic pin dimensions, we first checked the stability in a single high gain amplifier chain. Secondly, we had put two amplifier chains side by side, but 
running in opposite direction, and determined the improvement of the isolation between them by using the gap waveguide packaging technique. For the two cases, the proposed PMC or gap waveguide packaging technique were compared with conventional metal cavities with RF absorber and via based packaging. The schematic of the two test cases are shown in fig.4. The measured results for these two test cases are shown in fig. 5. From the single chain selfoscillation measurement results presented in fig. 5(a) and 5(b), we found no oscillation peaks for gap waveguide packaging (pin lid packaging) even after $65 \sim 70 \mathrm{~dB}$ total gain. On the other hand, for traditional metal wall packaging with absorbing material, resonance peaks appeared at around $40 \mathrm{~dB}$ of total forward gain.
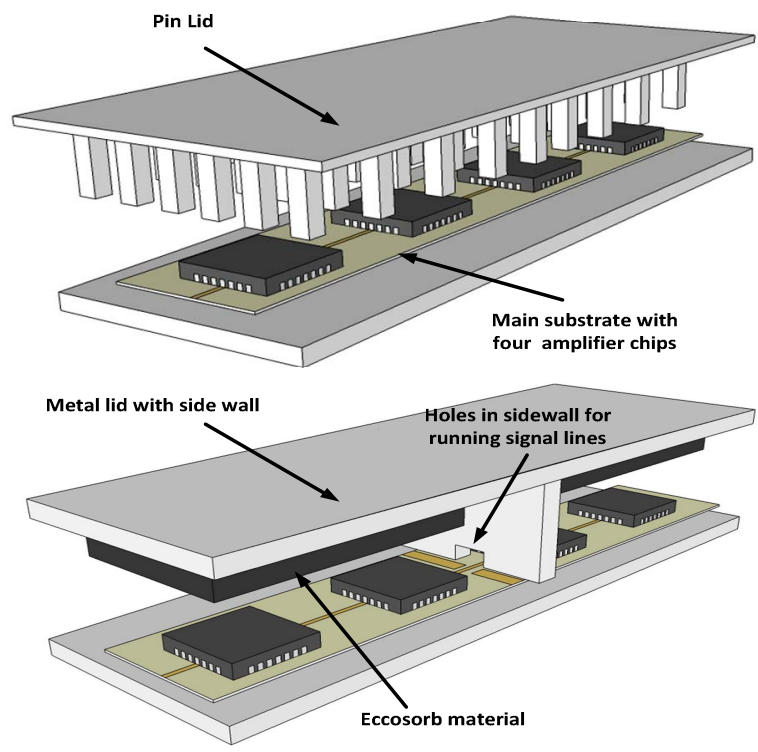

Fig.4 (a) Test circuit for isolation evaluation of a single amplifier chain (side walls not shown).
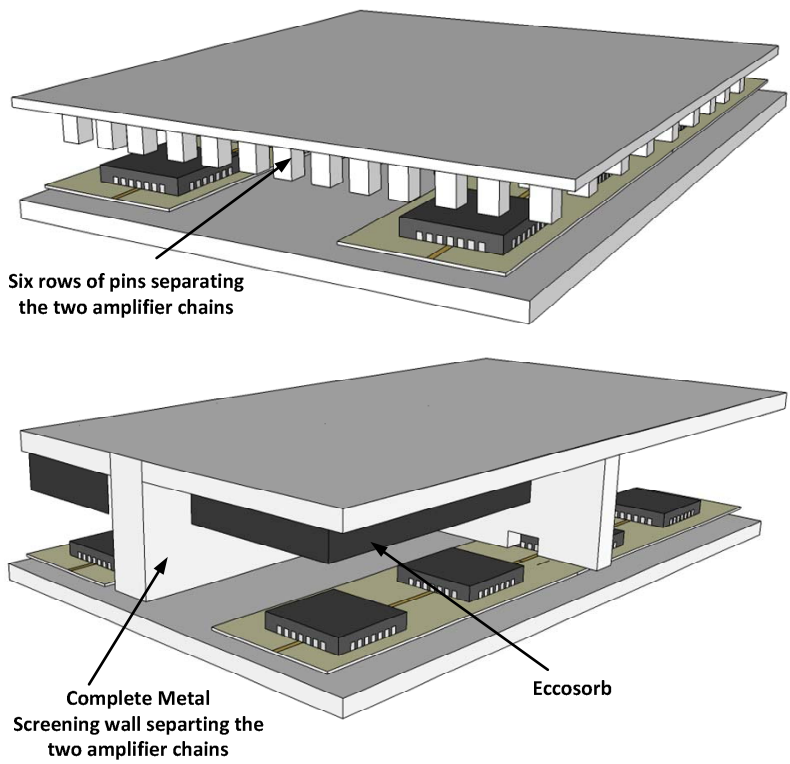

Fig.4(b) Test circuit for isolation evaluation of side by side amplifier chain (side walls not shown).
For the test case of two amplifier chains placed side by side, the measurement results showed that an isolation round $70 \mathrm{~dB}$ in average was achieved for a six pin row lid with isolation value ranging from $64 \mathrm{~dB}$ to $91 \mathrm{~dB}$. Similar isolation trend was observed also for complete metal wall shielding case. For the case of eight rows of pins, better isolation was observed.

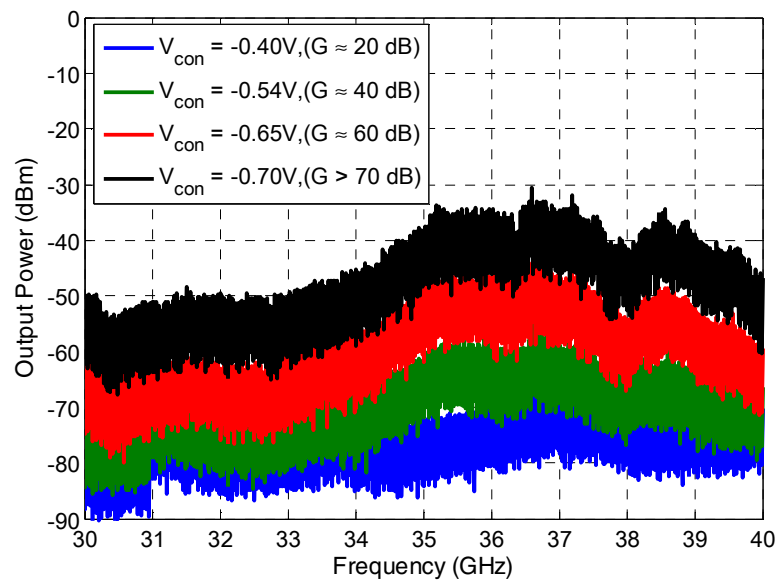

Fig.5 (a) Self-oscillation analysis for single amplifier chain packaged with pin lid.

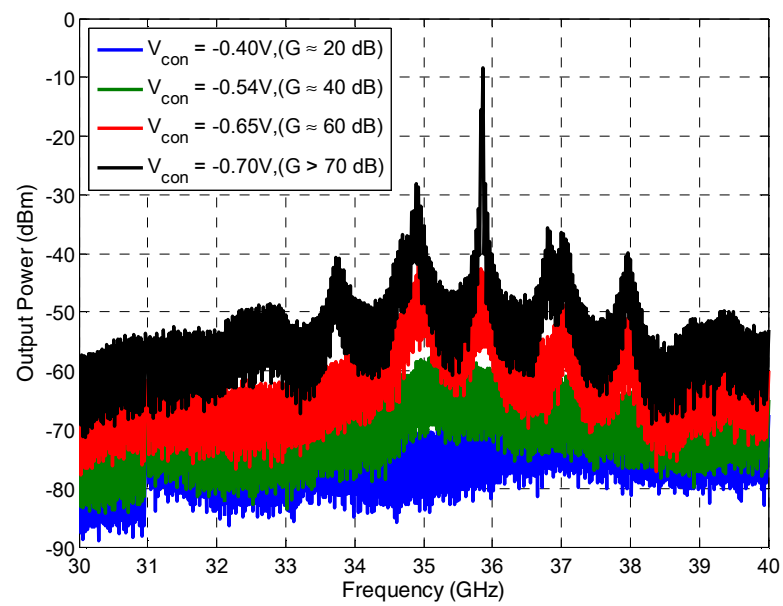

Fig.5 (b) Self-oscillation analysis for single amplifier chain packaged with pin metal lid and absorber

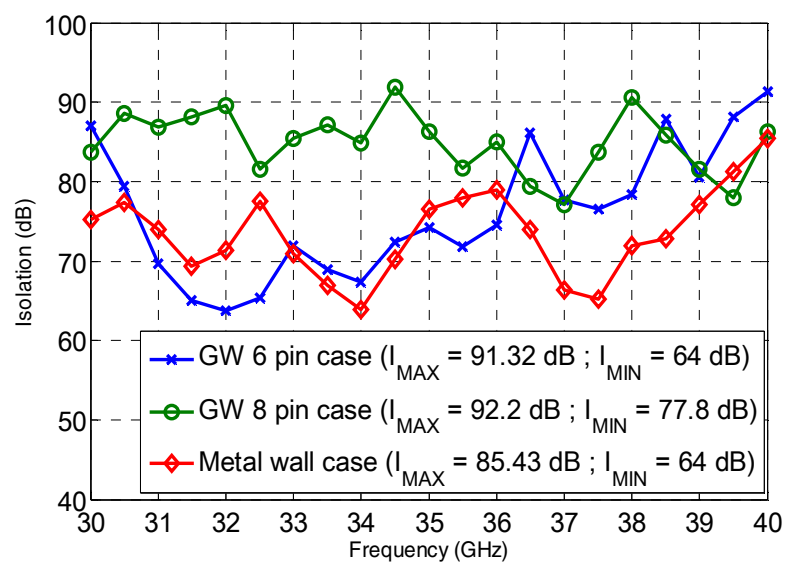

Fig.5(c) Isolation between two closely spaced amplifier chains. 
The details of the above mentioned active amplifier chain packaging work is presented in [21]. The manufactured prototypes for this work are shown in fig.6.
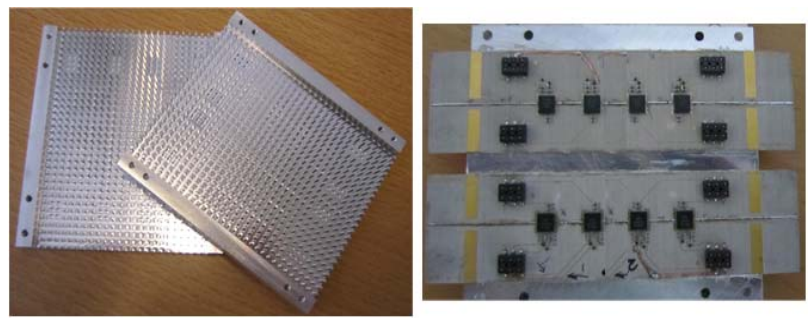

Two pin lids
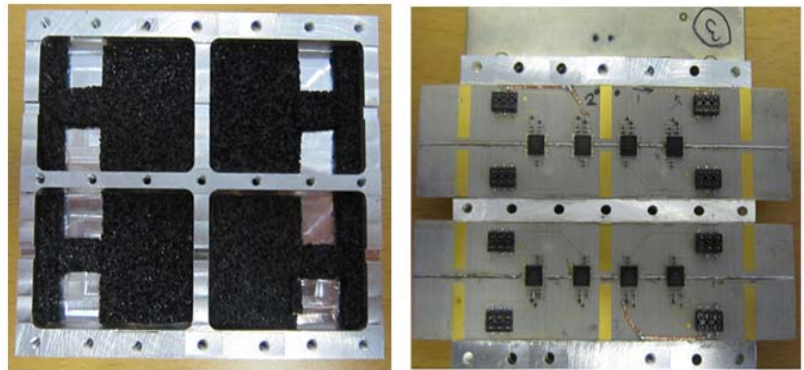

Metal wall with Eccosorb

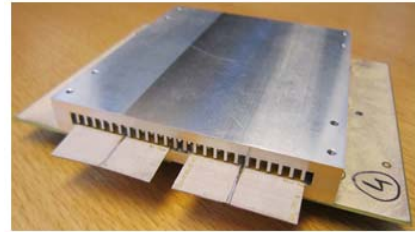

Top side ( mounted with Pin lid)

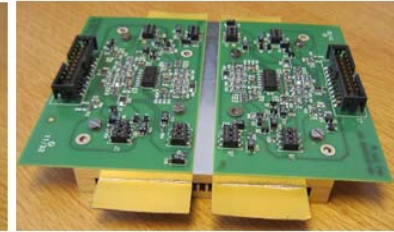

Bottom side ( Bias circuitry)
Fig.6 Pictures of the amplifier chain test circuits.

\section{LOW FREQUENCY GAP WAVEGUIDE PACKAGING SOLUTIONS}

The solution presented so far is very suitable for high frequency range but become very bulky if we try to implement for the low microwave range. The periodicity of pins can be as small as desired but their height is not so flexible and must be close to a $\lambda / 4$ size which is too big for low frequencies. A possible solution to this issue is to use springs instead of pins [22], i.e., the total length of the pin is very reduced by bending it as shown in Fig. 7. In the designed example from [22] the total height $\mathrm{h}$ was reduced to only $0.068 \lambda$ and also the periodicity is very small in terms of wavelength. The structure behaviour is very similar to the case of pins, with the main difference that here the influence of the gap $g$ in the bandwidth of the structure is very small. The reason is that in this case there is almost no capacitive effect with the upper plate when compared to the pins case. Still the bandwidth is close to $2: 1$ for this structure. The proposed structure is a very good solution for low frequency due to compactness but also because is made only with metal. However it is clear that its manufacturing is not easy and this can mean an increase in the cost. Another solution inspired in the same idea of bending pins was proposed in [23]. In this case the pins were replaced by 'zigzag' printed elements as presented in Fig. 8. The achieved compactness is not as good as for the springs but the manufacturing cost is considerably reduced. On the other hand, as this solution is intended for low frequencies, the use of dielectric materials is not a problem.
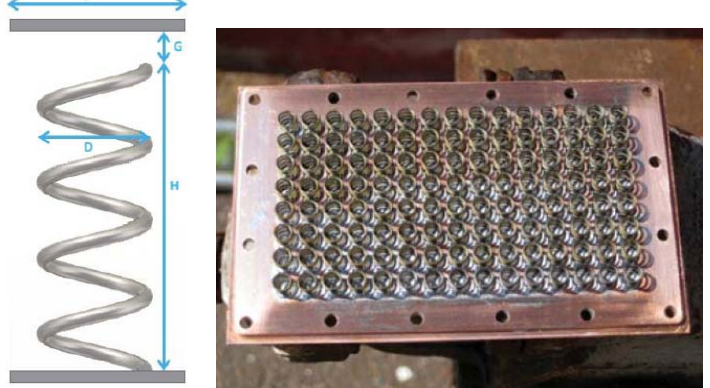

Fig.7 Solution for low frequency: 'the bed of springs'; Unit cell and manufactured prototype.

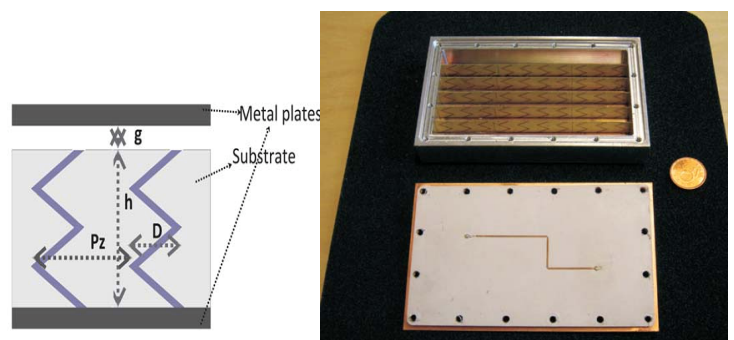

Fig.8 Solution for low frequency: printed zigzag lid; Unit cell and manufactured prototype.

In the example proposed in [23] we have used a height $h$ of $10 \mathrm{~mm}$, which is still low profile for the frequency range under study from 3 to $6 \mathrm{GHz}$. Again in this case the capacitive effect with the upper lid is almost negligible. This fact of weak dependency of the stop band with the gap size is of interest to integrate components, i.e., if required the gap size can be modified in some part of the structure and still the performance will be good. We have experimentally verified the good performances of these packaging solutions by measuring the transmission of a packaged double bent microstrip line as the one in the picture in Fig. 8. The line is measured with the proposed lid, with a smooth metal lid and also unpackaged. The results are presented in Fig. 9 for the two proposed geometries. It is clear how they removed the cavity modes of the structure and also that the use of a dielectric to print the zigzags do not introduce meaningful losses.

\section{CONCLUSION}

In this work, an overview of the newly proposed packaging or shielding technique based on gap waveguide technology has been discussed. The proposed packaging technology has the following advantages: scalable with frequency, independent of lateral mechanical cavity dimensions, improved insertion loss of the overall circuitry. Only, drawback of the proposed gap waveguide packaging technique is that - it operates over a specific bandwidth which can be up to an octave bandwidth. However, this proposed concept is particularly useful for active microwave components such as 


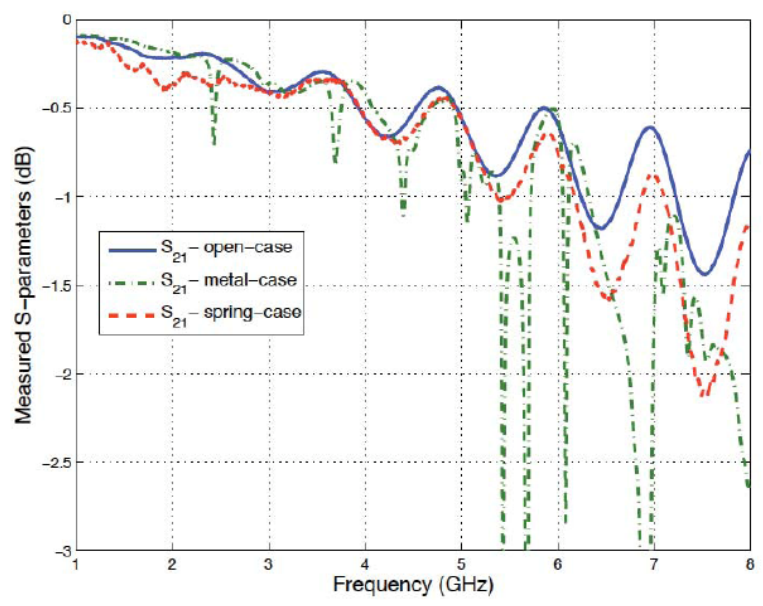

Fig.9(a) Measured $S_{21}$ parameters for a double bent microstrip line packaged with the lid of springs Comparison with smooth metal lid is also included.

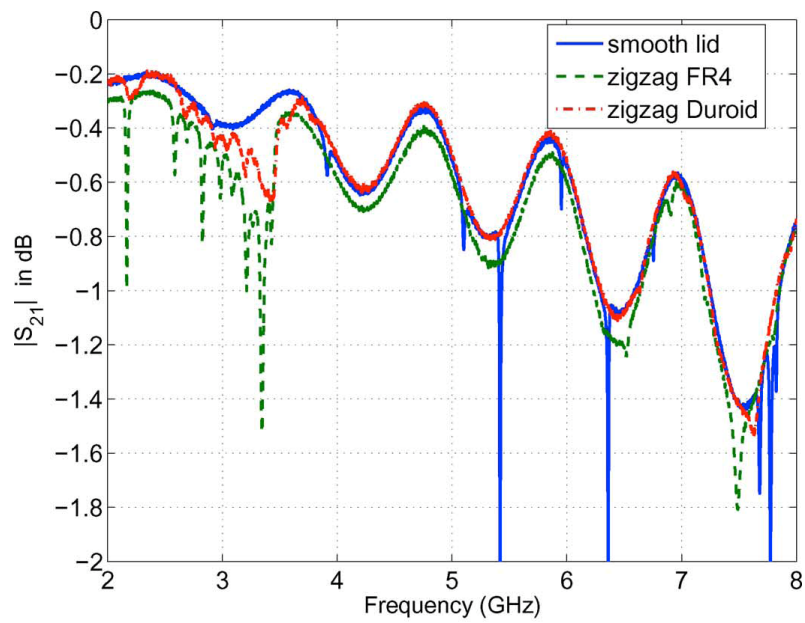

Fig.9(b) Measured $\mathrm{S}_{21}$ parameters for a double bent microstrip line packaged with the lid of zigzags. Comparison with smooth metal lid is also included.

high gain amplifier chains where package feedback problems, over the air coupling and coupling via substrate modes can play a critical role in cross-talk or signal integrity issue and may drive the amplifier chain towards unwanted oscillation. Apart from the high frequency solutions, we have presented also simple low frequency packaging solutions in this work.

\section{REFERENCES}

[1] R. Tummala and J. Laskar, "Gigabit wireless: System-on-a-package technology," Proc. IEEE, vol. 92, pp. 376-387, Feb. 2004.

[2] K. Wu, Y. J. Cheng, T. Djerafi, and W. Hong, "Substrate-integrated millimeter-wave and terahertz antenna technology," Proc. IEEE, vol. 100, no. 7, pp. 2219-2232, Jul. 2012.

[3] V. Prasad Kodali, "Engineering Electromagnetic Compatibility: Principles, Measurements and Technologies," ISBN:0-7803-1117-5, IEEE press, 1996.

[4] A.Margomenos, K.J.Herrick, M.I.Herman, S.Valas, and L. P.B.Katehi, "Isolation in Three-Dimensional Integrated Circuits," IEEE Trans. Micro. Theory and Techniques, vol. 51, no. 1, pp. 25-32, January 2003

[5] L.Schulwitz, S.Ortiz, and A. Mortazawi, "Packaging Method for Increased Isolation Using a Mcirostrip to Waveguide Transition, " IEEE Microwave and Wireless Component Letters, vol. 17, no. 3, pp. 163-165, March 2007.
[6] D. f. Williams, "Damping of the Resonant Modes of a Rectangular Metal Package," IEEE Transection on Microwave Theory and Techniques, vol. 37, no. 1, pp. 253-256, January 1989.

[7] W.H. Haydl, "On the Use of Vias in Conductor-Backed Coplanar Circuits," IEEE Trans. on Microwave Theory and Technique, vol. 50, no. 6, pp. 1571-1577, June 2002.

[8] P.-S. Kildal, A. Uz Zaman, E. Rajo-Iglesias, E. Alfonso and A. ValeroNogueira, "Design and experimental verification of ridge gap waveguides in bed of nails for parallel plate mode suppression," IET Microwave, Antennas \& Propagation, vol. 5, no. 3, pp. 262-270, March 2011.

[9] A. Uz. Zaman, P.-S. Kildal, M. Ferndahl and A. Kishk, "Validation of Ridge Gap Waveguide Performance Using in-house TRL Calibration Kit," 4th European Conference on Antennas and Propagation, Barcelona, April 2010.

[10] A.Valero-Nogueira, E.Alfonso, J. I. Herranz and P.-S. Kildal, "Experimental demonstration of local quasi-TEM gap modes in singlehard-wall waveguides," IEEE Microwave and Wireless Components Letters, vol. 19, no. 9, pp. 536-538, September 2009.

[11] P.-S. Kildal, "Artificially soft and hard surfaces in electromagnetics," IEEE Trans. Antennas Propag., vol. 28, no. 10, pp. 1537-1544, Oct.1990.

[12] F. Mesa, A. A. Oliner, D. R. Jackson, and M. J. Freire, "The influence of a top cover on the leakage from microstrip line," IEEE Trans. Microwave Theory and Techniques, vol. 48, no. 12, pp. 2240-2248, December 2000.

[13] W.E.McKinzie and N.G. Alexopoulos, "Leakage losses for the dominant mode of conductor-backed coplanar waveguide," IEEE Microwave Guided Wave Letter, vol. 2, pp. 65-66, February 1992.

[14] E. Alfonso, A Uz Zaman and P.-S. Kildal,“Ka-Band Gap Waveguide Coupled-Resonator Filter for Diplexer Application," IEEE Transactions on Comp., Packaging and Manufac. Technology, Vol.3, No-5, pp- 870879, May, 2013.

[15] A Uz Zaman and P.-S. Kildal, "Wide-band Slot Antenna Arrays with Single-layer Corporate-Feed Network in Ridge Gap Waveguide Technology," accepted for publication in IEEE Trans. Antennas Propag.

[16] S. Rahiminejad, A.U. Zaman, E. Pucci, H. Raza, V. Vassilev, S. Haasl, P. Lundgren, P.-S. Kildal and P. Enoksson, "Micromachined ridge gap waveguide and resonator for millimeter-wave applications," Elsevier, Sensors and Actuators A: Physical, vol. 186, pp. 264-269, October 2012.

[17] E. Rajo-Iglesias, A. Uz Zaman,and P.-S. Kildal, "Parallel plate cavity mode suppression in microstrip circuit packages using a lid of nails," IEEE Microwave and Wireless Components Letters, vol. 20, no. 1, pp. 31-33, December 2009.

[18] A.Uz. Zaman, J.Yang, and P.-S. Kildal, "Using Lid of Pins for Packaging of Microstrip Board for Descrambling the Ports of Eleven Antenna for Radio Telescope Applications," IEEE Antennas and Propagation Society International Symposium, July 2010.

[19] A. Algaba Brazález, A. Uz Zaman, and P.-S. Kildal, "Improved Microstrip filters Using PMC Packaging by Lid of Nails," IEEE Trans. Component,Packaging and Manufacturing Technology, vol. 2, no. 7, pp. 1075-1084, July 2012.

[20] A. Kishk, A. Uz Zaman,and P.-S. Kildal, "Numerical Prepackaging with PMC lid - Efficient and Simple Design Procedure for Microstrip Circuits including the Packaging," ACES Applied Computational Society journa, vol. 27, no. 5, pp. 389-398, May 2012.

[21] A. Uz Zaman, M.Alexanderson,T.Vukusic and P.-S. Kildal, "Gap Waveguide PMC Packaging Method for Improved Isolation of Circuit Elements in High Frequency Microwave Modules," IEEE Trans. On Comp., Packaging and Manufacturing Technology, vol.4, no.1, pp-1625, January 2014.

[22] E. Rajo-Iglesias, P.-S. Kildal, A.U. Zaman, A. Kishk, "Bed of Springs for Packaging of Microstrip Circuits in the Microwave Frequency Range," IEEE Transactions on Components, Packaging and Manufacturing Technology, , vol.2, no.10, pp.1623,1628, Oct. 2012.

[23] E. Rajo-Iglesias, E. Pucci, A.A. Kishk, Kildal, P.-S. "Suppression of Parallel Plate Modes in Low Frequency Microstrip Circuit Packages Using Lid of Printed Zigzag Wires," IEEE Microwave and Wireless Components Letters, , vol.23, no.7, pp.359,361, July 2013. 